\title{
Cistadenoma mucinoso del apéndice cecal como causa de gangrena intestinal*
}

\author{
Drs. HUMBERTO OSNAYA M. ${ }^{1}$, TAHITIANA ABELINA ZARAGOZA S. ${ }^{1}$, \\ MARCO ANTONIO MONDRAGÓN C. ${ }^{1}$
}

Servicio de Cirugía General del Centro Médico Lic. Adolfo López Mateos de Toluca Edo. de México. México.

\begin{abstract}
Appendiceal mucocele causing large bowel gangrene

Introduction: Appendiceal mucocele are lesions located in the cecum, which are found in 0.2 to $0.3 \%$ of appendectomies and correspond to $8-10 \%$ of all appendiceal tumors. Clinical case: We report a 83 years old female consulting for abdominal pain lasting 24 hours. On physical examination, signs of peritoneal irritation were found. The patient was operated, finding an appendiceal tumor that was coiled up in the mesentery with large bowel gangrene. An intestinal resection, terminal ileostomy, appendectomy and surgical lavage were performed. The pathological study of the surgical piece reported a mucinous cystadenoma.
\end{abstract}

Key words: Appendix, tumors, bowel gangrene.

\section{Resumen}

Introducción: Los tumores del Apéndice Cecal corresponden al 0,5\% de las neoplasias gastrointestinales y se encuentran entre el $0,8 \%$ y el $1 \%$ de las apendicectomías. El término Mucocele Apendicular (AM), se refiere a un grupo de lesiones localizados a nivel del ciego cuyo rango de incidencia está entre el $0,2 \%$ y el $0,3 \%$ de todas las apendicectomías, y entre el $8-10 \%$ de todos los tumores apendiculares. Las presentaciones clínicas más frecuentes son: cuadro clínico de apendicitis aguda, como hallazgo incidental como en el caso de esta paciente, o como masa abdominal en fosa ilíaca derecha. Caso clínico: Se presenta un caso de Cistadenoma Mucinoso Apendicular (CAM) encontrado como hallazgo incidental en un abdomen agudo quirúrgico con la particularidad de presentarse con una necrosis intestinal.

Palabras clave: Apendicitis aguda, cistoadenoma mucinoso, necrosis intestinal, cistoadenoma apendicular, gangrena intestinal.

*Recibido el 18 de junio de 2012 y aceptado para publicación el 24 de septiembre de 2012.

Los autores no refieren conflictos de interés.

Correspondencia: Dra. Tahitiana Abelina Zaragoza S.

Calle Tlatelolco \# 633 Colonia Guadalupe, Morelia Michoacán, México. tatis10018@hotmail.com 


\section{Caso clínico}

Paciente femenina de 83 años de edad con antecedente de hipertensión de larga evolución. Inicia su padecimiento $24 \mathrm{~h}$ previas, caracterizadas por dolor abdominal tipo cólico de gran intensidad, localizado en epigastrio y mesogastrio, distensión abdominal así como náuseas y vómitos en 10 ocasiones, sin especificar características. A la exploración física con palidez de tegumentos, mucosas orales deshidratadas, ruidos cardíacos rítmicos, aumentados en frecuencia, campos pulmonares sin compromiso pleuropulmonar, abdomen distendido, dolor generalizado a la palpación media y profunda, peristalsis disminuida, con signos de irritación peritoneal además de datos francos de respuesta inflamatoria sistémica. Se interviene quirúrgicamente con la sospecha diagnóstica de probable apendicitis aguda o trombosis mesentérica. Se realiza laparotomía exploradora reportando como hallazgos: Hernia interna estrangulada por tumoración apendicular enrollada en mesenterio, gangrena intestinal con segmento afectado de 1,5 $\mathrm{m}$ a partir de 2,5 $\mathrm{m}$ de asa fija hasta válvula ileocecal, líquido libre. Se realiza resección intestinal, ileostomía terminal, cierre distal en bolsa de Hartmann, apendicectomía y aseo quirúrgico. Se envía pieza quirúrgica al Servicio de patología donde se reporta: Cistoadenoma Mucinoso.

\section{Discusión}

El término mucocele es un término descriptivo macroscópico que hace referencia a una dilatación quística de la luz del apéndice ileocecal de etiología obstructiva, que produce un acumulo de material mucoide y se debe nominar en función de alguno de los procesos histopatológicos causales ${ }^{1-3}$. Las tumoraciones del apéndice son patologías poco frecuentes, su diagnóstico prequirúrgico es difícil por lo que la mayoría de estas patologías se intervienen con diagnóstico de apendicitis aguda y se encuentran como hallazgo incidental, dicha presentación caracterizada por la paciente presentada en este caso clínico $^{4-6}$. El tratamiento es quirúrgico pudiendo ser suficiente la apendicectomía sola en patologías benignas, o bien, tratamientos más invasivos como la hemicolectomía derecha y quimioterapia ${ }^{7,8}$ en sospecha de patologías malignas; siendo la resección intestinal e ileostomía el tratamiento sugerido para nuestro caso sin tener evidencia de histopatológico causal, dadas las condiciones de presentación de nuestro caso y la gangrena evidenciada ${ }^{9-12}$. La literatura refiere un predominio del sexo femenino, tal y como ocurre con nuestra paciente y una edad entre la quinta y sexta década en promedio ${ }^{13}$, sin embargo,

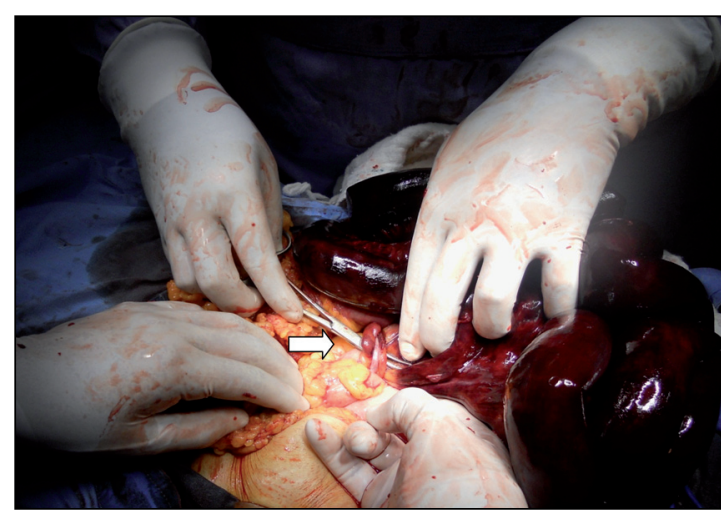

Figura 1. Apéndice cecal formando anillo de constricción de mesenterio.

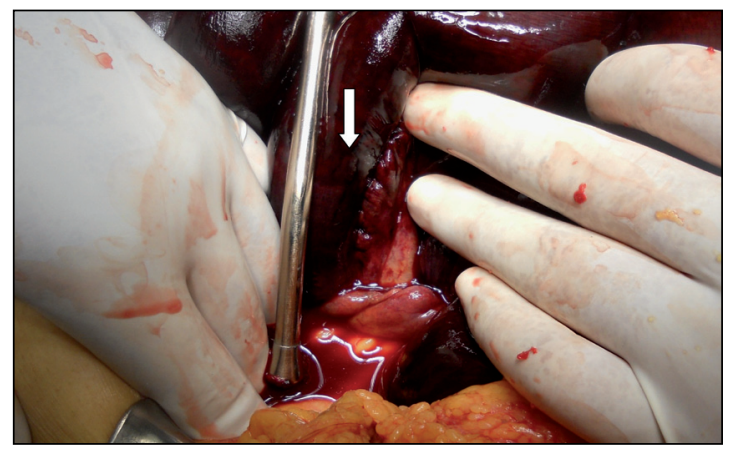

Figura 2. Gangrena Intestinal secundario a anillo de constricción de apéndice cecal.

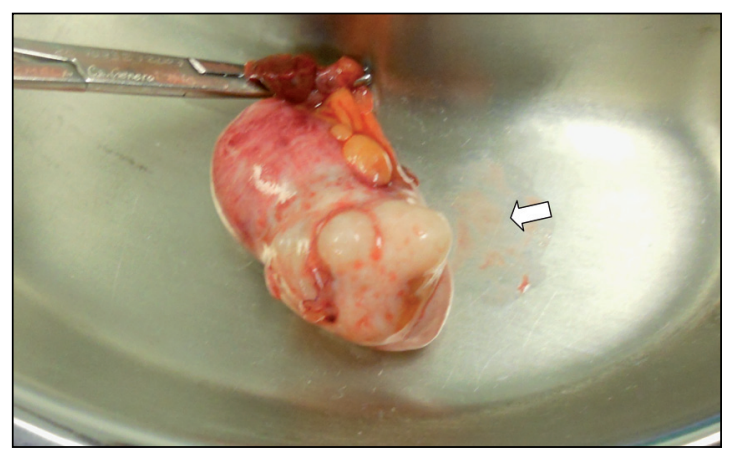

Figura 3. Cistadenoma en tercio distal de apéndice.

en diversas comunicaciones hay discordancia en estos datos ${ }^{14,15}$. Los CAM pueden ser tumores que alcanzan gran tamaño causando sintomatología secundaria a la compresión o desplazamiento de órganos y estructuras, o bien, la sintomatología puede ser inespecífica e incluso pueden permanecer asintomáticos hasta en el $25 \%$ de los pacientes. La manifestación más común es el dolor en fosa iliaca derecha que puede simular un cuadro de apendicitis 
aguda y si al cistoadenoma mucinoso se agrega una superinfección, pueden ser clínicamente indistinguibles ${ }^{16,17}$. Otras formas de manifestarse son, una masa palpable hasta en el 50\% y el resto suele encontrarse como un hallazgo incidental. Otra forma de presentación es la secundaria a una complicación como: hemorragia, invaginación intestinal, obstrucción intestinal, síntomas genitourinarios, torsión, gangrena, etc. En el caso de esta paciente su particularidad de este tumor encontrado es haberse complicado con una gangrena intestinal, la cual no se sospecha y que causa impacto por la poca o casi nula literatura existente que describa la incidencia de este hallazgo en la bibliografía revisada. Los métodos diagnósticos incluyen: Radiografía simple de abdomen: El cual evidencia una masa de tejido en el cuadrante inferior derecho, con efecto de masa en el intestino adyacente. Ultrasonido: que muestra una lesión encapsulada quística y en ocasiones se observa el "signo de la piel de cebolla" el cual consiste en muchas capas ecogénicas a lo largo de un apéndice dilatado ${ }^{18}$. Tomografía: con alta sensibilidad para la detección de los adenomas mucinosos (AM) ${ }^{19}$ en que se observa una imagen homogénea y quística bien delimitada, con áreas centrales de atenuación y contenido con densidad similar al agua ${ }^{20}$. En el $50 \%$ de los casos hay calcificaciones en la pared apendicular, muy sugestiva de mucocele ${ }^{21}$. Colonoscopia: Se observa la imagen característica de "signo de volcán" en la cual se aprecia un orificio central por el cual sale moco. Aun, y a pesar del arsenal de métodos diagnósticos, en el caso de esta paciente se decide la intervención quirúrgica dado el cuadro clínico tan florido de un abdomen agudo quirúrgico sin tener la clara sospecha de un cistadenoma mucinoso ${ }^{22}$. Laboratorio: El antígeno carcinoembrionario puede estar elevado en los mucoceles neoplásicos y puede ser usado para el seguimiento después de la resección ${ }^{23-25}$. El patólogo debe hacer un estudio exhaustivo buscando perforaciones inadvertidas que pueden cambiar el pronóstico, ya que los pacientes con un mucocele benigno o simple tienen un excelente pronóstico con tasa de supervivencia de cinco años del 91 al $100 \%$. Los pacientes con Pseudomixoma peritoneal tienen una tasa de supervivencia a cinco años de 25 a $65 \%$. Los pacientes con AM tienen seis veces mayor probabilidad de desarrollar un adenocarcinoma de colon, así como desarrollar posteriormente pseudomixoma peritoneal, por lo tanto, debe haber un seguimiento adecuado por esta alta incidencia de neoplasias sincrónicas o metacrónicas ${ }^{26,27}$. De la revisión bibliográfica y las controversias existentes podemos concluir que las neoplasias mucinosas del apéndice y las enfermedades peritoneales asociadas representan una de las áreas más confusas y controvertidas en la patología gastrointestinal debido a que se usan criterios histológicos inconsistentes para su diagnóstico y terminología descriptiva para su clasificación y el nulo reporte de complicaciones esperadas de acuerdo a su evolución como abdomen agudo. Por ello, consideramos debe crearse una revisión más amplia y estudio más amplio de una serie de casos de manejo y terapéutica actual.

\section{Referencias}

1. Aho A, Heinonen R, Lauren P: Benign, Malignant mucocele of the appendix: Histologic tipes and prognosis. Acta Chir Scand. 1973;139:392-400.

2. Serrano PA, Pérez-Bedmar JA, Larrañaga E. Mucocele apendicular. Revisión de la literatura y aportación de ocho casos. Rev Esp Enf Ap Digest. 1989;76:35-41.

3. Persaud T, Giant Mucinous Cystadenoma of the Appendix. RadioGraphics 2007;27:553-7.

4. Higa E, Rosai J, Pizzimbono CA, Wise L. Mucosal hiperplasia, mucinous Cystadenoma, And mucinous cystadenoma of appendix. A re-evaluation of the appendiceal mucocele. Cancer 1973;32:1525-41.

5. Sierra Montenegro E. Cistoadenoma Mucinoso de Apéndice. Informe de un Caso. Cir. 2010;78:257-260.

6. García García M.A. Cistoadenoma Mucinoso Apendicular. Actas Urol Esp. 2002;26:139-42.

7. Labastida M. Cistoadenocarcinoma Mucinoso de Apéndice Cecal. Presentación de un caso. INFORMED 2010;12:511-4.

8. Sugarbaker PH. The natural history, gross pathology, and histopathology of appendiceal epithelial neoplasms. EJSO 2002;32:644-7.

9. Caracappa D. Appendiceal mucocele. A case report and literature review. Ann Ital Chir. 2011;82:239-45.

10. Ingo Honnef. Appendiceal Mucinous Cystadenoma. RadioGraphics 2008;28:1524-7.

11. Lim HK, Lee WJ, Kim SH, Kim B, Cho JM, Byun JY. Primary mucinous cystadenocarcinoma of the appendix: CT findings. AJR Am J Roentgenol. 1999;173:1071-4.

12. Butte B. Jean Michel. Tumores Del Apéndice Cecal, Análisis antomoclínico y evaluación de la sobrevida alejada. Rev Chil Cir. 2007;59:217-22.

13. Tovío Almanza. Cáncer de Apéndice: incidencia en los pacientes atendido en el servicio de urgencias del hospital universitario del Caribe entre enero de 2007 y julio de 2009. Rev Cienc Biomed.2010;1:168-72.

14. Ruiz-Tovar J. Mucocele of the appendix. World J Surg. 2007;31:542-8.

15. Yacan S. A retrospective study on mucocele of the appendix presented with acute abdomen or acute appendicitis. J Emerg Med.(Hong Kong) 2011;18:144-9.

16. Roberge RJ, Park AJ. Mucocele of the appendix: an important clinical rarity. J Emerg Med. ( Hong Kong) 2006;30:303-6.

17. Caspi B, Cassif E, Auslender R, Herman A, Hagay Z, Appleman $\mathrm{Z}$. The onion skin sign: a specific sonogra- 
phic marker of appendiceal mucocele. J Ultrasound Med. 2004;23:117-21.

18. Kim-Fuchs C. Apendiceal mucocele in an elderly patient: how much surgery? Case Rep Gastroenterol. 2011;5:516-22.

19. Federle MP, Anne VS. Mucocele of the appendix. In: Federle MP, ed. Diagnostic imaging: abdomen. Salt Lake City, Utah: Amirsys, 2004;26-27.

20. Honnef I, Moschopulos M, Roeren T. Appendiceal mucinous cystadenoma. Radiographics 2008;28:1524-7.

21. Hamilton DL, Stormont JM. The volcano sign of appendiceal mucocele. Gastrointest Endosc. 1989;35:453-6.

22. Rampone B. Giant appendiceal mucocele: report of a case and brief review. World J. Gastroenterol.
2005;11:476-3.

23. González-Moreno S, Sugarbaker PH: Right hemicolectomy does nor confer a survival advantage in patients with mucinous carcinoma of the appendix and peritoneal seeding. Br J Surg. 2004;91;304-11.

24. Ki Bum Park. Single-incision Laparoscopic Surgery for Appendiceal Mucoceles: Safety and Feasibility in a Series of 16 Consecutives Cases. J Korean Soc Coloproctol. 2011;27:287-92.

25. Stocchi L, Bruce G, Dirk R, Jeff R. Surgical treatment of appendiceal mucocele. Arch Surg. 2003;138:585-90.

26. Rappoport J, Steiner M, Moyano L, Amat J, Bezama J, Garrido R, et al. Mucocele apendicular. Rev. Chil Cir. 2002;54:339-44. 